\title{
Surfactant replacement therapy in ARDS: white knight or noise in the system?
}

\author{
Terence E Nicholas, Ian R Doyle, Andrew D Bersten
}

\begin{abstract}
Although one would predict that surfactant replacement therapy would be effective in acute respiratory distress syndrome (ARDS), a recent large trial proved unsuccessful, possibly reflecting the nature of the surfactant used. Given the importance of the unique proteins in the action of surfactant, these would seem vital components of any exogenous surfactant. The ability to identify patients at risk of ARDS and to characterise their surfactant might allow prophylactic treatment with a nebulised, complementary, tailor-made preparation of surfactant. Advanced cases might undergo bronchoscopic focal lavage to remove plasma proteins and inflammatory mediators prior to focal instillation of surfactant to areas of greatest need. Ventilation regimens might be adjusted both to minimise trauma and to conserve endogenous surfactant.
\end{abstract}

(Thorax 1997;52:195-197)

Keywords: surfactant, ARDS, surfactant replacement therapy, surfactant associated proteins.

Surfactant is a complex mixture of phospholipids, neutral lipids, and unique surfactant proteins (SP-A, B, C) which lines the alveolar gas-liquid interface where it continuously turns over with a half life measured in hours. Surfactant both reduces and allows surface tension to vary directly with the radius of curvature of the alveolus, hence reducing the work of breathing, stabilising the lung, and maintaining fluid balance. In addition, it has antiglue, host defence, antioxidant, and anti-inflammatory properties.

In the mid 1960s Chu et al performed an ambitious trial with surfactant replacement therapy (SRT) in premature infants. ${ }^{1}$ Not only did the outcome suggest that such therapy may have a negative effect in respiratory distress syndrome (RDS), but it indicated that pulmonary ischaemia was the primary event which then resulted in surfactant deficiency. With hindsight, the main flaw of the study was the assumption that dipalmitoylphosphatidylcholine (DPPC) was the only necessary component of surfactant. A second flaw was using a nebuliser which, at that stage, delivered little to the alveolar compartment. Both focus on the importance of the appropriate animal model studies. Indeed, it was another five years before Enhorning and Robertson successfully instilled mature rabbit surfactant to rescue premature pups. ${ }^{2}$ SRT is now used routinely in neonatal intensive care units where it has reduced the mortality of infants under 29 weeks gestation by as much as $50 \%{ }^{3}$ By contrast, Anzueto et al recently reported that aerosolised Exosurf had no benefit in a multicentre trial involving 364 patients with sepsis induced ARDS. ${ }^{4}$ This has engendered a certain déjà vu among students of surfactant history, remembering the trial of Chu et al. Since patients with ARDS appear to be prime candidates for SRT, why did this recent trial produce negative results? Alternatively, are we on the wrong track, and any consideration of SRT in ARDS is nothing more than "noise in the system"?

Scientific basis

ARDS, with a mortality of $40-60 \%$, remains one of the most frustrating and common conditions confronting the critical care physician. Whereas neonatal RDS is clearly a disease of prematurity characterised by surfactant deficiency, the pathophysiology of ARDS is far more complex, involving a variety of insults leading to neutrophil infiltration, pulmonary fibrosis, and increased alveolocapillary permeability. However, animal studies have shown that, regardless of the initial insult, compromising surfactant initiates a vicious cycle, encouraging oedema and atelectasis, which further compromises surfactant.

In ARDS surfactant may be compromised in several ways. The increase in permeability results in plasma proteins entering the alveolar hypophase and inhibiting the surface properties of surfactant. Alternatively, the insult may directly damage the alveolar type II cells and decrease the synthesis, release, or reprocessing of surfactant (fig 1). Proteases and reactive oxygen species in the hypophase may affect SP-A, B and C and so interfere with the alveolar processing of surfactant which normally leads to the formation of an active monolayer (fig 1). Surfactant from patients with established ARDS contains decreased levels of SP-A, SP-B, phosphatidylcholine and phosphatidylglycerol, and increased levels of other phospholipids. 


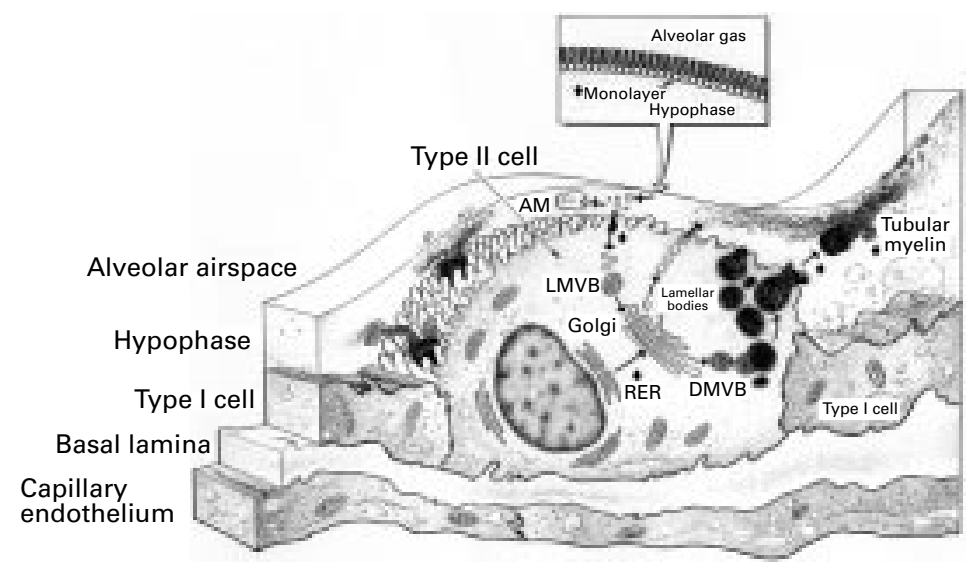

Figure 1 An alveolar type II cell showing two surfactant release mechanisms: lamellar bodies and the constitutive release of surfactant protein- $A$. Surfactant is shown being both phagocytosed by an alveolar macrophage ( $A M$; not to scale) and being taken back into the cell and reutilised. The inset illustrates the monolayer at low lung volume, comprising almost pure dipalmitoylphosphatidylcholine which, in the gel (solid) phase, is able to withstand very large lateral forces, literally "splinting" open the alveolus. The thickness of the hypophase has been exaggerated, as it normally averages $0.14 \mu \mathrm{m}$ over the flat sections and $0.89 \mu \mathrm{m}$ at the wall junctions. RER = rough endoplasmic reticulum; $L M V B=$ light multivesicular body; DMVB= dense multivesicular body. * indicates possible sites of damage in ARDS; these include the synthesis, release and reuptake of surfactant, and the formation of tubular myelin and of the monomolecular layer.

Importantly, some of these changes are apparent in "at risk" patients. ${ }^{5}$ We need more information relating surfactant composition (possibly even including SP-A isoforms) in the individual patient to the initial insult, the inspired oxygen, and the ventilatory regimen.

CHOICE OF SURFACTANT

Exosurf is a protein-free preparation which combines DPPC with tyloxapol and hexadecanol as emulsifiers and spreading agents. Given the vital role of the surfactant proteins in overcoming the surfactant inactivating properties of plasma proteins in maintaining the surface active, large, tubular myelin-rich aggregates of surfactant which then form the active monolayers, one must conclude that Exosurf was a poor choice. Indeed, Exosurf is only effective in about $50 \%$ of neonates with RDS ${ }^{6}$ possibly only those with sufficient endogenous surfactant proteins. As patients with ARDS are very susceptible to nosocomial pneumonia, the ideal exogenous surfactant might contain SP-A for its host defence capabilities, as well as for its ability to stimulate re-uptake of alveolar surfactant leading to increased de novo synthesis. Finally, SP-A and SP-B may reverse the abnormally low ratio of large to small surfactant aggregates found in ARDS. ${ }^{7}$

METHOD OF DELIVERY

In neonatal RDS the surfactant deficiency is relatively homogeneous so that a bolus of approximately $100 \mathrm{mg} / \mathrm{kg}$ surfactant has a homogeneous effect. A disadvantage of this approach in ARDS is the amount required, costing as much as \$US 70000 per patient. Whereas nebulisation is more economical, $<5 \%$ of surfactant reaches the lungs ${ }^{4}$ and is deposited in the better ventilated alveoli where it is least required. A different approach, albeit invasive, is to use a bronchoscope both to lavage and then to instil surfactant directly into the worst affected regions identified by computed tomographic scanning. This would tend to remove from the alveolus mediators which potentiate lung injury while minimising the dose of surfactant.

\section{MODE OF VENTILATION}

The pattern of breathing clearly affects surfactant homeostasis and composition. ${ }^{89}$ Surfactant is released from alveolar type II cells by a single large breath, and both alveolar surfactant and the flux between the tubular myelin-rich and poor alveolar fractions (fig 1) are proportional to tidal volume $(\mathrm{VT}){ }^{8}$ Release at any peak inspired pressure can be reduced by increasing the end expired pressure (EEP), suggesting that less is required to maintain a viable alveolus at an increased functional residual capacity. ${ }^{8}$ The approach using high EEP/low VT with occasional large breaths appears to benefit the patient with $\mathrm{ARDS}^{10}$ and is consistent with surfactant being released by distortion of the type II cell. ${ }^{8}$ The rate of reuptake of surfactant is related to the rate of release rather than to the amount in the alveolus, and both can be increased by $\beta$ adrenoceptor agonist drugs. ${ }^{8}$ Finally, increasing VT also increases the rate of synthesis of surfactant phospholipids. ${ }^{8}$ It may therefore be possible to titrate ventilation in order to minimise the alveolar surfactant required and actually alter its composition ${ }^{9}$ while increasing synthesis and turnover pharmacologically. The damage due to high inspired oxygen and volumes might be minimised by monitoring serum SP-A and SP-B as indices of alveolocapillary permeability. ${ }^{11} 12$

\section{Therapeutic potential}

Patients considered "at risk" would be identified from serum levels of SP-A and SP-B, ${ }^{12}$ their surfactant composition determined from tracheal aspirates, and complementary preparations of synthetic phospholipids and surfactant proteins formulated. Patients would then be treated prophylactically by aerosol at a stage when their lungs are still ventilating relatively normally. This would be economical, provide a homogeneous distribution, prevent the vicious cycle, and allow the lung time to repair. In patients with overt ARDS focal lavage and surfactant instillation via a bronchoscope would be complemented with a low VT/high EEP ventilation regimen, possibly incorporating permissive hypercapnia, ${ }^{10}$ thus minimising the need for surfactant while avoiding the risk of barotrauma. $\beta$ adrenoceptor agonist drugs might be included in the instillate to increase surfactant turnover, resolve oedema, and increase mucociliary transport. Finally, it is unreasonable to expect SRT to reverse end stage ARDS with widespread destruction of the alveolocapillary membrane. Possibly these patients would benefit from liquid ventilation. 
1 Chu J, Clements JA, Cotton EK, Klaus MH, Sweet AY, Thomas $\mathrm{M}$, et al. Neonatal pulmonary ischemia: clinical and physiologic studies. Pediatrics 1967;40:709-82.

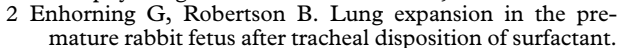
Pediatrics 1972;50:55-6.

3 Jobe AH. Pulmonary surfactant therapy. N Engl F Med 1993; 328:861-8.

4 Anzueto A, Baughman RP, Guntupalli KK, Weg JG, Weidemann HP, Raventos AA, et al. Aerosolized surfactant in adults with sepsis-induced acute respiratory distress in adults with sepsis-induced acute respirat

5 Gregory TJ, Longmore WJ, Moxley MA, Whitsett JA, Reed CR, Fowler AA, et al. Surfactant chemical composition and biophysical activity in acute respiratory distress syndrome. $\mathcal{F}$ Clin Invest 1991;88:1976-81.

6 Jobe AH. Surfactant in the perinatal period. Early Hum Dev 1992;29:57-62.

7 Veldhuizen RAW, McCraig LA, Akino T, Lewis JF. Pulmonary surfactant subfractions in patients with the acute respiratory distress syndrome $A m \mathcal{F}$ Respir Crit Care Med 1995;152:1867-71

8 Nicholas TE. Control of turnover of alveolar surfactant NIPS 1993;8:12-18.

9 Doyle IR, Jones ME, Barr HA, Orgeig S, Crockett AJ, McDonald CF, et al. Composition of human pulmonary surfactant varies with exercise and level of fitness. $A m$ Respir Crit Care Med 1994;149:1619-27.

10 Amato MBP, Barbas CSV, Medeiros DM, Schettino GPP, Filho GL, Kairalla RA, et al. Beneficial effects of the "open lung approach" with low distending pressures in acute respiratory distress syndrome. Am $\mathcal{F}$ Respir Crit Care Med 1995;152:1835-46.

11 Doyle IR, Nicholas TE, Bersten AD. Serum surfactant protein-A levels in patients with acute cardiogenic pulmonary edema and adult respiratory distress syndrome. Am $\mathcal{f}$ Respir Crit Care Med 1995;152:307-17.

12 Doyle IR, Bersten AD, Nicholas TE. Surfactant proteins (SP-A and B) in plasma in critically ill patients with respiratory disease. Am 7 Respir Crit Care Med 1995;151: A73. 